\title{
An Iterative Procedure for Removing Random-Valued Impulse Noise
}

\author{
Raymond $\mathrm{H}$. Chan, Chen $\mathrm{Hu}$, and Mila Nikolova
}

\begin{abstract}
This paper proposes a two-stage iterative method for removing random-valued impulse noise. In the first phase, we use the adaptive center-weighted median filter to identify pixels which are likely to be corrupted by noise (noise candidates). In the second phase, these noise candidates are restored using a detail-preserving regularization method which allows edges and noise-free pixels to be preserved. These two phases are applied alternatively. Simulation results indicate that the proposed method is significantly better than those using just nonlinear filters or regularization only.
\end{abstract}

Index Terms-Adaptive center-weighted median filter, impulse noise, regularization methods.

\section{INTRODUCTION}

I MAGES are frequently corrupted by impulse noise due to noisy sensors or channel transmission errors [10]. There are many types of impulse noise. Let $Y_{i j}$ be the gray level of a true image $\mathbf{Y}$ at pixel location $(i, j)$ and $\left[n_{\min }, n_{\max }\right]$ be the dynamic range of $\mathbf{Y}$. Let $X_{i j}$ be the gray level of the noisy image $\mathbf{X}$ at pixel $(i, j)$, then

$$
X_{i j}= \begin{cases}R_{i j}, & \text { with probability } r \\ Y_{i j}, & \text { with probability } 1-r\end{cases}
$$

where $R_{i j} \in\left[n_{\min }, n_{\max }\right]$ are random numbers and $r$ is the noise ratio. For example, for fixed-valued (salt-and-pepper) impulse noise, noisy pixels $X_{i j}$ take either $n_{\min }$ or $n_{\max }$, see [14]. In this paper, we focus on general random-valued impulse noise, where $R_{i j}$ can be any numbers between $n_{\min }$ and $n_{\max }$ (see [8]). Cleaning such noise is far more difficult than cleaning fixed-valued impulse noise, since for the latter, the differences in gray levels between a noisy pixel and its noise-free neighbors are significant most of the times.

The main approach for removing impulse noise is to use median-based filters (see, e.g., [1], [13], and [16]). However, since filters typically are implemented invariantly across the images, they also tend to modify pixels that are not affected by noise. In addition, when the noise ratio is high, they are prone to edge jitter, and that the details and edges of the original image are usually blurred by the filters [18].

Manuscript received December 12, 2003; revised April 28, 2004. This work was supported by HKRGC Grant CUHK4243/01P and CUHK DAG 206022. The associate editor coordinating the review of this manuscript and approving it for publication was Dr. Steven L. Grant.

R. H. Chan and C. Hu are with the Department of Mathematics, The Chinese University of Hong Kong, Shatin, NT, Hong Kong (e-mail: rchan@math.cuhk.edu.hk; chu@math.cuhk.edu.hk).

M. Nikolova is with Centre de Mathematiques et de Leurs Applications (CNRS UMR 8536), ENS de Cachan, 94235 Cachan Cedex, France (e-mail: nikolova@cmla.ens-cachan.fr).

Digital Object Identifier 10.1109/LSP.2004.838190
To improve performance, various decision-based filters have been proposed where possible noise pixels are first identified and then replaced by using the median filter. Examples of decision-based filters are the center-weighted median filter [15], the adaptive center-weighted median filter (ACWMF) [9], the adaptive median filter [14] and the median filter based on homogeneity information [19]. These filters are good in locating the noise, even in a high noise ratio. However, the main drawback is that the replacement of the noisy pixels by the median filter entails blurring of details and edges, especially when the noise ratio is high.

Recently, a detail-preserving variational method (DPVM) has been proposed to restore impulse noise [17]. It uses a nonsmooth data-fitting term (e.g., $\ell_{1}$ ) along with edge-preserving regularization. In this paper, we propose to combine ACWMF with DPVM for restoring images that are highly corrupted by random-valued impulse noise. Our method involves two steps which are applied alternatively. First, noisy pixels are detected using ACWMF; then these pixels are selectively restored by DPVM. Since in each iteration the edges and the details are preserved for the noise candidates by the regularization method, and no changes are made to the signal candidates, the performance of this combined method is much better than just using either ACWMF or DPVM, especially when the noise ratio is high. Our method can restore large patches of noisy pixels because it introduces pertinent prior information via the regularization term. It is most efficient to deal with high noise ratio, e.g., ratio as high as $50 \%$.

The outline of the paper is as follows. In Section II, we review ACWMF. Our denoising scheme is given in Section III. In Section IV, we demonstrate the effectiveness of our method using various images.

\section{REVIEW OF ACWMF}

ACWMF is a good method for removing random-valued impulse noise when the noise ratio is not high—see [9] or Figs. 1(b) and 2(b) in Section IV. Here, we give a brief review of the filter.

Let the window size be $(2 h+1)^{2}$ and $L=2 h(h+1)$. Denote by $X_{i j}$ the gray level of the noisy image at pixel location $(i, j)$. Let

$$
Y_{i j}^{2 k}=\operatorname{median}\left\{X_{i-u, j-v},(2 k) \diamond X_{i j} \mid-h \leq u, v \leq h\right\}
$$

where $2 k$ is the weight given to pixel $(i, j)$, and $\diamond$ represents the repetition operation. Clearly, $Y_{i j}^{0}$ is the output of the standard median filter, whereas $Y_{i j}^{2 k}$ is the output of the identity filter when $k \geq L$. We define the differences

$$
d_{k}=\left|Y_{i j}^{2 k}-X_{i j}\right|
$$




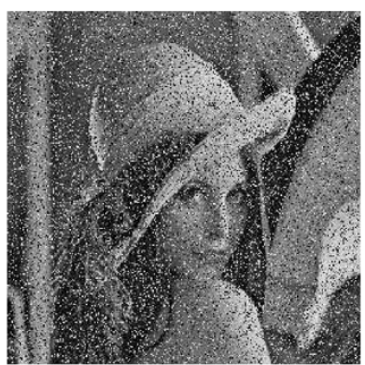

(a)

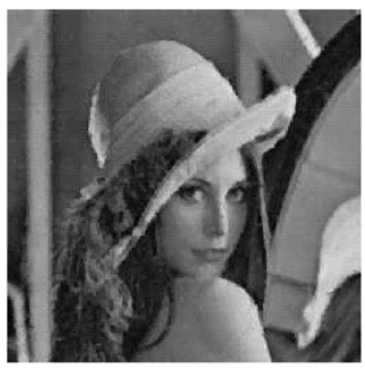

(c)

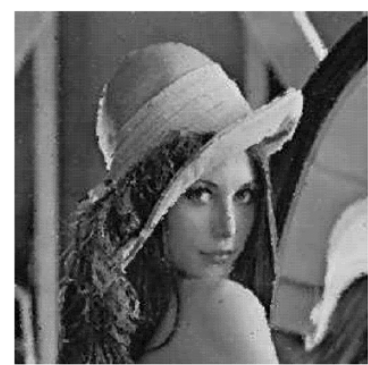

(b)

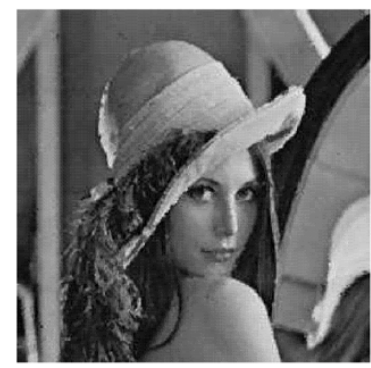

(d)

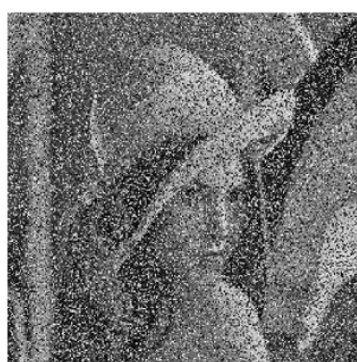

(a)

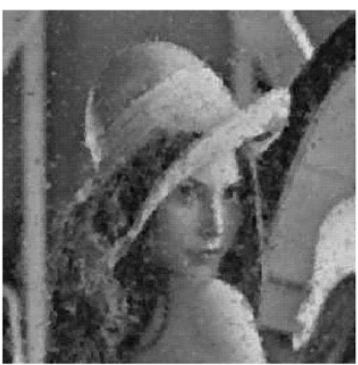

(c)

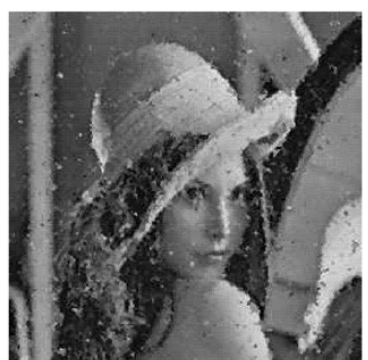

(b)

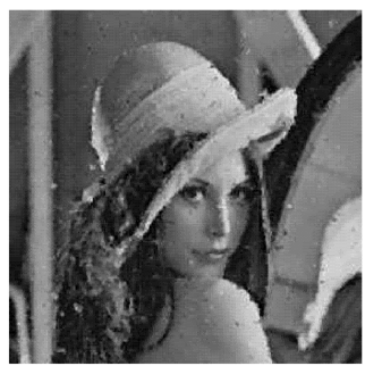

(d)
Fig. 1. (a) Image with $30 \%$ noise. Restored images by (b) ACWMF with $s=$ 0.6 , (c) DPVM with $\beta=0.19$, and (d) our method with $\beta=2, s=0.6$ and four iterations.

where $k=0,1, \ldots, L-1$. It is readily seen that $d_{k} \leq d_{k-1}$ for $k \geq 1$ (see [7]).

To determine whether the current pixel $(i, j)$ is corrupted, a set of thresholds $T_{k}$ are employed, where $T_{k-1}>T_{k}$ for $k=1,2, \ldots, L-1$. If any one of the inequalities $d_{k}>T_{k}, k=$ $0,1, \ldots, L-1$ is true, then $X_{i j}$ is regarded as a noise candidate and replaced by the median, i.e., $Y_{i j}^{0}$. Otherwise, $X_{i j}$ is regarded as a signal candidate and will not be changed.

If $3 \times 3$ windows are used (i.e., $h=1$ and $L=4$ ), four thresholds $T_{k}, k=0, \ldots, 3$, are needed. The median of the absolute deviations from the median (MAD), which is defined as

$$
\text { MAD }=\text { median }\left\{\left|X_{i-u, j-v}-Y_{i j}^{0}\right|:-h \leq u, v \leq h\right\}
$$

is a robust estimate of dispersion [12], [2] and its scaled forms are used as the thresholds. Specifically, one sets

$$
T_{k}=s \cdot \mathrm{MAD}+\delta_{k}, \quad 0 \leq k \leq 3
$$

with

$$
\left[\delta_{0}, \delta_{1}, \delta_{2}, \delta_{3}\right]=[40,25,10,5]
$$

and $0 \leq s \leq 0.6$ (see [9]). This choice yields satisfactory results in filtering random-valued impulse noise when the noise ratio is not high [see Fig. 1(b)]. However, for a high-level noise ratio, the filter cannot preserve the fine features in the images [see Fig. 2(b)].

\section{OUR METHOD}

When the noise ratio is high, ACWMF may falsely detect some noise-free pixels as noisy pixels. If these erroneous noise candidates form patches, and are located near to edges, DPVM
Fig. 2. (a) Image with 50\% noise. Restored images by (b) ACWMF with $s=$ 0.3 , (c) DPVM with $\beta=0.19$, and (d) our method with $\beta=2.3, s=0.1$ and four iterations.

will distort them. To alleviate the problem, we apply our method iteratively with different thresholds. More precisely, at the early iterations, we take large thresholds in ACWMF so that it will only select pixels that are most likely to be noisy. Then we restore them by DPVM. In the subsequent iterations, we decrease the thresholds to include more noise candidates. Since the edges and the details are preserved by the regularization successfully in each iteration, the restored image will not be distorted by the method.

In the following we give our algorithm. Let $\mathcal{V}_{i j}$ be the set of the four closest neighbors of $(i, j)$, not including $(i, j)$.

\section{Algorithm}

1) Set $r=0$. Initialize $\mathbf{X}^{(r)}$ to be the observed image.

2) Apply ACWMF with the thresholds $T_{k}^{(r)}, 0 \leq k \leq 3$, to the image $\mathbf{X}^{(r)}$ to get the noise candidate set $\mathcal{M}^{(r)}$.

3) Let $\mathcal{N}^{(r)}=\bigcup_{l=0}^{r} \mathcal{M}^{(l)}$.

4) For all $(i, j) \notin \mathcal{N}^{(r)}$, take $\hat{Y}_{i j}=X_{i j}^{(r)}$.

Restore all pixels in $\mathcal{N}^{(r)}$ by minimizing the following functional over $\mathcal{N}^{(r)}$ :

$$
\begin{aligned}
f(\mathbf{Y})= & \sum_{(i, j) \in \mathcal{N}^{(r)}}\left\{\left|Y_{i j}-X_{i j}^{(r)}\right|\right. \\
& +\frac{\beta}{2}\left(\sum_{(m, n) \in \mathcal{V}_{i j} \cap \mathcal{N}^{(r)}} \varphi\left(Y_{i j}-Y_{m n}\right)\right. \\
& \left.\left.+\sum_{(m, n) \in \mathcal{V}_{i j} \backslash \mathcal{N}(r)} \varphi\left(X_{m n}^{(r)}-Y_{i j}\right)\right)\right\}
\end{aligned}
$$

where $\varphi$ is an edge-preserving potential function. Notice that $\mathcal{V}_{i j} \backslash \mathcal{N}^{(r)}$ is composed of those neighbors of $(i, j)$ which at step $r$ have been detected as signal candidates. 
TABLE I

ERRORS OF RESTORED IMAGES AT 30\% NOISE

\begin{tabular}{c|c|c|c|c|c|c} 
& & bird & bridge & camera & goldhill & lena \\
\hline \multirow{4}{*}{ PSNR } & Noise Image & 15.85 & 13.98 & 13.79 & 15.23 & 14.48 \\
\cline { 2 - 7 } & ACWMF & 32.06 & 22.21 & 24.35 & 26.57 & 27.18 \\
& DPVM & 33.26 & 22.44 & 24.72 & 27.13 & 27.29 \\
& Our method & 33.72 & 22.76 & 25.08 & 27.52 & 28.33 \\
\hline \multirow{5}{*}{ MAE } & Noise Image & 18.48 & 23.10 & 23.45 & 19.95 & 21.63 \\
\cline { 2 - 7 } & ACWMF & 1.61 & 8.43 & 4.17 & 4.36 & 3.32 \\
& DPVM & 2.25 & 11.90 & 6.06 & 6.18 & 4.97 \\
& Our method & 1.27 & 7.95 & 3.67 & 3.85 & 2.80
\end{tabular}

The minimizer $\hat{\mathbf{Y}}$ of (4) is obtained by using the algorithm presented in [17], but restricted to $\mathcal{N}^{(r)}$.

5) Set $\mathbf{X}^{(r+1)}=\hat{\mathbf{Y}}$.

6) If $r<r_{\max }$, set $r=r+1$ and go back to Step 2 .

Possible choices for $\varphi$ in Step 4 are

$$
\begin{aligned}
\varphi(t) & =\sqrt{\alpha+t^{2}}, \quad \alpha>0 \\
\varphi(t) & =|t|^{\alpha}, \quad 1<\alpha \leq 2 \\
\varphi(t) & = \begin{cases}\frac{\alpha t^{2}}{2}, & \text { if }|t| \leq \frac{1}{\alpha}, \quad \alpha>0 \\
|t|-\frac{1}{2 \alpha}, & \text { if }|t|>\frac{1}{\alpha},\end{cases}
\end{aligned}
$$

(see [11], [5], [4], and [6]). In Step 2, we use $3 \times 3$ windows and thresholds of the form

$$
T_{k}^{(r)}=s \cdot \mathrm{MAD}^{(r)}+\delta_{k}+20\left(r_{\max }-r\right)
$$

for $0 \leq k \leq 3,0 \leq r \leq r_{\max }$, and $0 \leq s \leq 0.6$, cf. (1)-(3). In practice, four iterations are enough, i.e., $r_{\max }=3$ and the output is $\mathbf{X}^{(4)}$.

\section{Simulations}

In this section, we compare our method with ACWMF [9] and DPVM [17]. The 256-by-256 picture of Lena is used as the true image. Then $30 \%$ and $50 \%$ of the pixels are corrupted by random noise uniformly distributed on its dynamic range $\left[n_{\min }, n_{\max }\right]$ [see Figs. 1(a) and 2(a)]. Henceforth, we use the potential function $\varphi(t)=|t|^{1.3}$. In the simulations, for each noise level, the parameters $s$ in (2) and $\beta$ in (4) are chosen to give the best restoration in terms of peak-to-noise-ratio (PSNR) (see [3, p. 556]).

From Figs. 1-2, we see that there are noticeable noise patches in the images restored by either ACWMF or DPVM, especially when the noise ratio is 50\%. In contrast, our method has successfully suppressed the noise while preserving most of the details and the edges in both cases.

To assess the effectiveness of our method in processing various images, we tried four other 256-by-256 gray scale images. The parameters $s$ and $\beta$ were chosen to be the same as in the previous simulations. The results in terms of PSNR and the mean
TABLE II

ERRORS OF RESTORED IMAGES AT 50\% NOISE

\begin{tabular}{c|c|c|c|c|c|c} 
& & bird & bridge & camera & goldhill & lena \\
\hline \multirow{4}{*}{ PSNR } & Noise Image & 13.62 & 11.82 & 11.59 & 12.99 & 12.28 \\
\cline { 2 - 7 } & ACWMF & 25.36 & 19.53 & 20.43 & 22.74 & 22.40 \\
& DPVM & 26.81 & 20.21 & 21.17 & 23.63 & 23.08 \\
& Our method & 29.93 & 20.77 & 22.53 & 25.04 & 25.48 \\
\hline \multirow{5}{*}{ MAE } & Noise Image & 30.91 & 38.18 & 39.00 & 33.22 & 36.04 \\
\cline { 2 - 7 } & ACWMF & 4.96 & 14.87 & 9.88 & 9.05 & 8.26 \\
& DPVM & 6.22 & 16.92 & 12.12 & 10.88 & 10.20 \\
& Our method & 2.84 & 12.84 & 6.86 & 6.85 & 5.41
\end{tabular}

absolute error (MAE) (see [3, p. 556]), are summarized in Tables I and II. From the tables, we see that our method are significantly better than the other two methods. ${ }^{1}$ Overall, our restored images are significantly better than those restored by the other two methods.

We end by considering the complexity of our algorithm. Since $r_{\max }=3$, the algorithm requires four applications of ACWMF and four applications of DPVM restricted to the set of the noisy pixels $\mathcal{N}^{(r)}$. Like other medium-type filters, ACWMF can be done very fast. The application of DPVM is the most time-consuming part as it requires the minimization of the functional in (4). For example, for $30 \%$ noise, our method takes 30 times more CPU time than ACWMF. The timing can be improved by better implementations of minimization routines for solving (4).

\section{REFERENCES}

[1] G. R. Arce and R. E. Foster, "Detail-preserving ranked-order based filters for image processing," IEEE Trans. Acoust., Speech, Signal Processing, vol. ASSP-37, pp. 83-98, Jan. 1987.

[2] J. Astola and P. Kuosmanen, Fundamentals of Nonlinear Digital Filtering. Boca Raton, FL: CRC, 1997.

[3] B. Barnett, Handbook of Image Processing, A. Bovik, Ed. New York: Academic, 2000.

[4] M. Black and A. Rangarajan, "On the unification of line process, outlier rejection, and robust statistics with applications to early vision," Int. J. Comput. Vis., vol. 19, pp. 57-91, July 1996.

[5] C. Bouman and K. Sauer, "A generalized Gaussian image model for edge-preserving MAP estimation," IEEE Trans. Image Processing, vol. 2, pp. 296-310, July 1993.

[6] P. Charbonnier, L. Blanc-Féraud, G. Aubert, and M. Barlaud, "Deterministic edge-preserving regularization in computed imaging," IEEE Trans. Image Processing, vol. 6, pp. 298-311, Feb. 1997.

[7] T. Chen, K.-K. Ma, and L.-H. Chen, "Tri-state median filter for image denoising," IEEE Trans. Image Processing, vol. 8, pp. 1834-1838, Dec. 1999.

[8] T. Chen and H. R. Wu, "Space variant median filters for the restoration of impulse noise corrupted images," IEEE Trans. Circuits Syst. II, vol. 48, pp. 784-789, Aug. 2001.

[9] — , "Adaptive impulse detection using center-weighted median filters," IEEE Signal Processing Lett., vol. 8, pp. 1-3, Jan. 2001.

[10] R. C. Gonzalez and R. E. Woods, Digital Image Processing, 2nd ed. Englewood Cliffs, NJ: Prentice-Hall, 2001.

[11] P. J. Green, "Bayesian reconstructions from emission tomography data using a modified EM algorithm," IEEE Trans. Med. Imag., vol. 9, pp. 84-93, Mar. 1990.

[12] F. R. Hampel, E. M. Ronchetti, P. J. Rousseeuw, and W. A. Stahel, Robust Statistics: The Approach Based on Influence Functions. New York: Wiley, 1986.

${ }^{1}$ Pictures of the noisy images and the restored images can be found at www.math.cuhk.edu.hk rchan/paper/chn/. 
[13] W.-Y. Han and J.-C. Lin, "Minimum-maximum exclusive mean (MMEM) filter to remove impulse noise from highly corrupted images," Electron. Lett., vol. 33, no. 2, pp. 124-125, 1997.

[14] H. Hwang and R. A. Haddad, "Adaptive median filters: New algorithms and results," IEEE Trans. Signal Processing, vol. 4, pp. 499-502, Apr. 1995.

[15] S.-J. Ko and Y. H. Lee, "Center weighted median filters and their applications to image enhancement," IEEE Trans. Circuits Syst., vol. 38, pp. 984-993, Sept. 1991.

[16] Y. H. Lee and S. A. Kassam, "Generalized median filtering and related nonlinear filtering techniques," IEEE Trans. Acoust., Speech, Signal Processing, vol. ASSP-33, pp. 672-683, June 1985.
[17] M. Nikolova, "A variational approach to remove outliers and impulse noise," J. Math. Imaging Vis., vol. 20, pp. 99-120, Jan. 2004.

[18] T. A. Nodes and N. C. Gallagher Jr., "The output distribution of median type filters," IEEE Trans. Commun., vol. COM-32, pp. 532-541, May 1984.

[19] G. Pok, J.-C. Liu, and A. S. Nair, "Selective removal of impulse noise based on homogeneity level information," IEEE Trans. Image Processing, vol. 12, pp. 85-92, Jan. 2003. 